\title{
Fatal Disseminated Cryptococcus Infection in an Immunocompetent Patient
}

\author{
Reza Hashemi ${ }^{1}$; Alireza Majidi ${ }^{2, *}$; Ali Tabatabaey ${ }^{2}$; Hassan Motamed $^{3}$ \\ ${ }^{1}$ Department of Pulmonary Medicine, Shahid Beheshti University of Medical Sciences, Tehran, IR Iran \\ ${ }^{2}$ Department of Emergency Medicine, Shahid Beheshti University of Medical Sciences, Tehran, IR Iran \\ 3 Department of Emergency Medicine, Ahvaz Jundishapur University of Medical Sciences, Ahvaz, IR Iran \\ ${ }^{*}$ Corresponding author:Alireza Majidi, Department of Emergency Medicine, Shahid Beheshti University of Medical Sciences, Shohadaye Tajrish Medical Center, Tajrish sq., Tehran, IR \\ Iran. Tel|Fax:+98 -2122721155, E-mail: alirezamajidi330@yahoo.com
}

Received: October 12, 2013; Accepted: February 26, 2014

\begin{abstract}
Introduction: Cryptococcosis is an opportunistic fungal infection mostly in immunocompromised patients. Here we present the immunocompetent case with crypotoccosis.

Case Presentation: A 60-year-old man without any history of immunodeficiency or major risk factor of fungal infection, referred to the emergency department with systemic symptoms and low back pain. The patient's condition gradually deteriorated within days by forming the clinical picture of a multi -system disease and eventually leading to a fatal outcome. The results of skin biopsy, BAL, gastrointestinal biopsy samples and post mortem lung and liver necropsies confirmed the disseminated Cryptococcus neoformans infection in smears and cultures.

Discussion: This case emphasizes the need for a high index of suspicion for fungal infections in patients with multi -organ involvements who are unresponsive to treatment. Even without any obvious history or sign of immunodeficiency.
\end{abstract}

Keywords:Cryptococcosis; Immunocompetence; Mycoses

\section{Introduction}

Cryptococcosis is considered as an opportunistic infection, typically in immunocompromised patients. This eukaryotic pathogen has two common strains including C. neoformanse and C. gattii , the pigeon droppings and eucalyptus trees are their environmental reservoirs, respectively (1). These are the only strains with the capacity to optimally grow at temperatures above $30^{\circ} \mathrm{C}$ giving them the ability to infect the human body (2). Both strains cause diseases in immunocompetent and immunosuppressed hosts (3). Here we present a case of disseminated cryptococcosis in a patient without any immune deficiency.

\section{Case Presentation}

A 60-year-old previously healthy man was admitted to the emergency department of Shohadaye Tajrish Hospital with a chief complaint of malaise and weakness. A month before his admission he had been treated for left lower extremity radicular pain with oral non-steroidal anti-inflammatory drugs (NSAIDs) and diazepam. The patient had sought medical attention at another center two weeks prior to admission in our ED, with a chief complaint of generalized weakness, fatigue, and anorexia. At the time, a previously unrecognized renal failure was diagnosed and the patient was discharged.

The patient was a mine worker without any history of smoking, alcohol consumption, illicit drug abuse, or unsafe sexual contact. He did not have any history of recent travel, blood transfusion, or animal contact. The patient's father had been treated for pulmonary tuberculosis. There was no complaint of visual symptoms, cough, dyspnea, fever, night sweats, or bowel habit changes, but a gradual weight loss within the past two to three months was recorded.

During the physical examination he was ill, lethargic, and confused. Initial vital signs were as follows: Blood pressure 95/50 mmHg, heart rate $100 \mathrm{bpm}$, Respiratory Rate $16 \mathrm{bpm}$ and his body temperature was $37^{\circ} \mathrm{C}$. Examination of the head and neck revealed a bitemporal atrophy. His ENT (Ear, Nose, and Throat) exams were normal except for dry mucosa. Clear lung fields and a II/VI systolic murmur in the apex was detected on cardiopulmonary auscultation. The abdomen was soft with normal bowl sounds. No lymphadenopathy was detected throughout the body. The nails were free of clubbing, cyanosis and edema, with normal symmetric pulses. Reduced skin turgor was detected and rehydration therapy was initiated. Initial ECG examination showed sinus tachycardia with normal axis, segments and intervals. The chest X-ray (Figure 1) showed a bilateral miliary pattern with few patchy consolidations, and a ground glass density in both of lower lobes, more evident in the right lung. The findings are presented in Table 1.

Copyright (C) 2014, Infectious Diseases and Tropical Medicine Research Center. This is an open-access article distributed under the terms of the Creative Commons Attribution License, which permits unrestricted use, distribution, and reproduction in any medium, provided the original work is properly cited. 


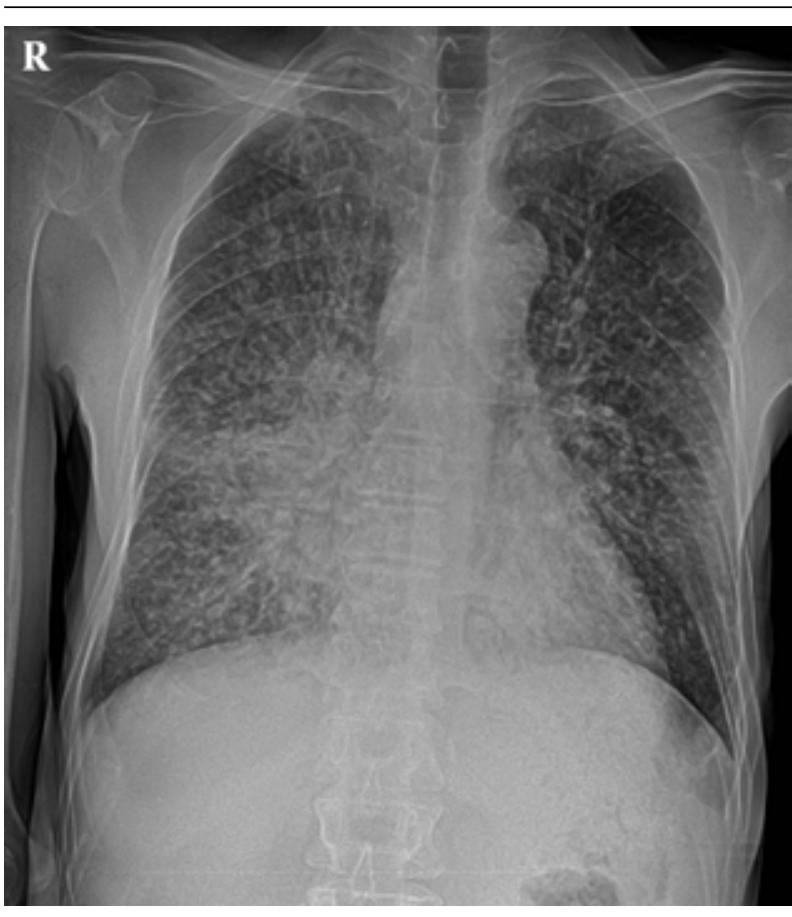

Figure 1. Chest X-Ray With Miliary Pattern



Echocardiography was done to rule out congestive heart failure and showed moderate mitral regurgitation, moderate tricuspid regurgitation, pulmonary hypertension with a pulmonary artery pressure of $36 \mathrm{mmHg}$, septal hypertrophy and a left heart ejection fraction of $65-70 \%$. Whole body scan was performed with the suspicion of undiagnosed myeloma and metastatic foci with an unknown source. It revealed high diffuse activity in axial skeleton, which was suggestive of metabolic bone disease causing low back pain. Electrophoresis of serum proteins also showed normal results.

Based on the results of the urinalysis and the new onset of renal failure, an ultrasonography of the urinary tract was prescribed and the right and left kidney size was 130 and $129 \mathrm{~mm}$ respectively, with an increased parenchymal echo. Bilateral hydronephrosis with mild distention of proximal ureters was also detected. A $10-\mathrm{mm}$ stone in the right middle calyx and an 8-mm stone in the left superior calyx detected. Daily BUN and creatinine was checked, which showed decreasing trends in both elements. On the 5th day of admission, the patient developed papular cutaneous lesions on his face and upper limbs, which gradually spread to the other parts of the body whichbecame umbilicated. After a dermatologist consult, skin biopsy was done, and IV acyclovir was administered with a presumptive diagnosis of Kaposi Varricelliform. On the 7th day of admission, the lesions on face and lumbar region became hemorrhagic and a new aphthus ulcer in the oral cavity was detected for which oral nistatin drops were administered.

Thoracic and abdomen pelvic CT -scans showed no pathologic findings. A few small calcified lymph nodes in the mediastinum with a diffuse nodular pattern in both lungs were detected. A blind broncoalveolar lavage (BAL) was carried out which drained a bloody specimen sent for serologic and pathologic evaluations. During the second week of admission, the patient had a limited episode of Upper GI bleeding without coagulopathy. UGI endoscopy showed pangasteroduodenitis and colonoscopy revealed colitis. Biopsy specimens were also obtained. On the 11th day of admission he developed a sudden loss of consciousness with neck rigidity. The patient withdrew from pain and manifested a rightward gaze. He was afebrile and no other focal neurologic deficits were detected. Brain CT scan without contrast revealed subarachnoid hemorrhage at the base of his skull. An attempt to perform lumbar puncture failed to retrieve CSF and repeated attempts were avoided due to the patient's critical condition. Anti Tuberculosis medication was added to antibiotics and antiviral treatment to cover viral hemorrhagic fever. He was intubated and mechanical ventilation was initiated.

Despite antibiotic, antiviral and anti -TB regimens the patient's condition continued to deteriorate. The patients BUN and creatinine levels dropped to $38 \mathrm{mg} / \mathrm{dL}$ and $1.4 \mathrm{mg} / \mathrm{dL}$ respectively. On the other hand the patient had hypernatremia and hypokalemia that were treated accordingly. Gradual onset of metabolic acidosis was also 
demonstrated by repeated $A B G$ analysis. Leukocytosis with a WBC of 11800 was detected on the 12th day of admission. One day later, apnea and cardiac arrest occurred and resuscitation was unsuccessful.

Results from skin biopsy, BAL, GI biopsy and post mortem lung and liver necropsy approved the presence of Cryptococcus neoformans in smears and cultures.

\section{Discussion}

For the first time in 1894, Cryptococcus was isolated from peach juice. It was brought back into attention in the era of immunosuppression due to AIDS and organ transplantation (1). People at higher risks of infections are those with HIV/AIDS especially when their CD4 level is less than $200 / \mathrm{m}^{3}$, recipients of solid organ transplants, and people with hematologic malignancies (3). Although, infections with milder forms of immunosuppression have infrequently been reported $(1,4)$, one study reported that even in HIV - uninfected patients with disseminated disease, an underlying condition associated with immunocompromise was found in $91 \%$ of cases. with liver cirrhosis, diabetes mellitus, and autoimmune diseases (5). This number has been estimated to be $78 \%$ in patients with pulmonary disease (6). On the other hand C. gattii has been associated with fatal disease in immunocompetent individuals (3). The patient presented here did not have a history of immune deficiency and no form of obvious immunodeficiency was disclosed during evaluations. A recently diagnosed chronic kidney disease was detected which can be attributed to fungal involvement of the urinary system. Furthermore the species causing the disease was less notorious C. neoformans.

Cryptococcosis is acquired by inhalation of fungal spores in to the lung. Once fungal spores find their way into the patient's respiratory tract, the disease will manifest. According to the severity of cryptococcal infection, and patient's health status, a wide range of clinical manifestations may be resulted including cough, fever, pneumonia, meningoencephalopathy, disseminated disease, or even death. In immunocompetent hosts, the pulmonary infection has a tendency toward spontaneous resolution and is frequently asymptomatic. If the immune system fails to overcome the infection at this point, organisms will spread, through blood to the brain and other tissues (1). Disseminated cryptococcosis is defined either by a positive culture of samples from at least two different sites, or a positive blood culture (5). In this case Cryptococcus was isolated from the skin, gastrointestinal, lung, and liver tissue samples, and the urine specimen was suspicious with limited yeast count and showed a mild increase of WBC and protein level.

Pulmonary cryptococcosis in patients with AIDS forms a disseminated thoracic disease with an interstitial lung pattern and lymph node enlargement; whereas the infection in immunocompetent hosts shows single or multiple nodular lesions or mass -like consolidations. Widespread miliary lung lesions have been also observed (7).
In this case, the patient had minimal respiratory symptoms with nodular, interstitial, and miliary disease on chest imaging. BAL analysis revealed cryptococcal colonization, but this was not a diagnostic finding (1).

In this case, the initial complaints were new onset of renal insufficiency and low back pain which are both very uncommon presentations for disseminated disease even in the immunosuppressed patients. Even after the recognition of pulmonary involvement, the family history of tuberculosis mislead the treatment team. This is not an uncommon mistake. In a study on 23 HIV - negative patients with pulmonary cryptococcosis, nine were initially diagnosed with tuberculosis, and six with metastatic disease (7), this issue emphases on the difficulty of diagnosis in such patients. In the presented patient here the first manifestation of disease was a low back pain which was attributed to vertebral involvement based on whole body scan findings. Bony involvement is a rare finding even in disseminated involvements of disease in immunosuppressed patients comprising only $5-10 \%$ of the cases (8). To our knowledge this is the first case of disseminated cryptococcosis in an immunocompetent patient with low back pain. Secondary involvement of skin is seen in $10 \%$ to $20 \%$ of immunocompromised patients with cryptococcosis. Lesions typically appear as pedunculated, dome -shaped papules with an umbilicated center, but cases of dermatitis and cellulitis have also been reported (9). In our case, skin involvement was indeed a secondary process occurred during hospital admission and predominantly manifested as umbilicated papules. Unfortunately our patient died before the diagnosis of cryptococcosis was confirmed and the patient did not receive timely antifungal treatments. It has been shown that delay in diagnosis may increase the mortality rate (6). Yet, even after diagnosis, the response to treatment is not guaranteed. Even with availability of highly active antiretroviral treatment, the mortality rate caused by acute cryptococcal meningoencephalitis within three months is around 20\% (10). Furthermore, at least one -third of patients with cryptococcal meningitis have shown mycologic and/or clinical failures (1).

In this case, with no history of immunodeficiency, an uncharacteristic initial manifestation of low back pain and renal insufficiency, and a positive family history of tuberculosis, all led to the delayed diagnosis of this fatal disseminated fungal disease. The initial workup aimed at finding the various causes, before the onset of pulmonary and neurologic symptoms. The fact that fungal infection was not initially suspected, led to the routine analysis of specimens for fungal infection which took seven to ten days. Therefore the results were prepared after the patient death. This case further emphasizes the need for a high index of suspicion for fungal infections in patients with multi -organinvolvementandunresponsivetotreatment, without any obvious history or sign of immunodeficiency.

\section{Authors' Contributions}

Reza Hashemi was responsible for the study concept 
and supervised the preparation and drafting of the paper. Alireza Majidi participated in drafting the manuscripts, administrative support, and its critical revision. Ali Tabatabaey contributed in data collection, preparation of the manuscript and contributed to important intellectual content. Hassan Motamed contributed in data collection and study concept.

\section{Funding/Support}

The study was funded by the authors' academic grants.

\section{References}

1. Li SS, Mody CH. Cryptococcus. Proc Am Thorac Soc. 2010;7(3):186-96.

2. Perfect JR. Cryptococcus neoformans: the yeast that likes it hot. FEMS Yeast Res. 2006;6(4):463-8.

3. La Hoz RM, Pappas PG. Cryptococcal infections: changing epidemiology and implications for therapy. Drugs. 2013;73(6):495-504.
4. Giri PP, Pal P, Gouda BR, Sinha R. Disseminated Cryptococcosis in a Patient With Nephrotic Syndrome. Arch Pediatr Infect Dis. 2014;3(2):E8932.

5. Chuang YM, Ho YC, Chang HT, Yu CJ, Yang PC, Hsueh PR. Disseminated cryptococcosis in HIV-uninfected patients. Eur J Clin Microbiol Infect Dis. 2008;27(4):307-10.

6. Pappas PG, Perfect JR, Cloud GA, Larsen RA, Pankey GA, Lancaster DJ, et al. Cryptococcosis in human immunodeficiency virus-negative patients in the era of effective azole therapy. Clin Infect Dis. 2001;33(5):690-9.

7. Song KD, Lee KS, Chung MP, Kwon OJ, Kim TS, Yi CA, et al. Pulmonary cryptococcosis: imaging findings in 23 non-AIDS patients. Korean J Radiol. 2010;11(4):407-16.

8. Singh R, Xess I. Multiple osseous involvements in a case of disseminated cryptococcosis. Indian J Orthop. 2010;44(3):336-8.

9. Lee JJ, Hsia RY. Cutaneous cryptococcal infection as a manifestation of disseminated disease. Cryptococcus neoformans. Ann Emerg Med. 2011;57(2):100.

10. Perfect JR, Dismukes WE, Dromer F, Goldman DL, Graybill JR, Hamill RJ, et al. Clinical practice guidelines for the management of cryptococcal disease: 2010 update by the infectious diseases society of america. Clin Infect Dis. 2010;50(3):291-322. 